\title{
Nutrition and Health Function of Soybean Drawing Protein and its
}

\section{Application in Food}

\author{
Xue-Jun LIU ${ }^{1, a}$, Xuan ZHANG ${ }^{1, b}$, Chang ZHU ${ }^{2, c}$, Yu XU ${ }^{1, d}$, Jia-Hui WANG ${ }^{1, e}$ \\ Xue-Jun LIU *
}

${ }^{1}$ College of Food Science and Engineering, Jilin Agricultural University, Changchun, Jilin, 130118,

China

\author{
${ }^{2}$ Jilin Engineering Vocational College, Food Engineering Branch, Siping, Jilin,136000, China \\ aliuxuejun63@163.com, b287863920@qq.com, 14170567@qq.com, d1422491537@qq.com, \\ e412491945@qq.com, *liuxuejun63@163.com
}

Keywords: Soybean drawing protein; Nutrition and health function; Application; A review

Abstract. In this review, soybean drawing protein was reviewed. The definition, nutrition and health function of soybean drawing protein and its application in food were detailed. And the future research directions of soybean drawing protein were outlooked, the article noted that the soybean drawing protein in the future should focus on researching on expanding the scope of application of soybean drawing protein and biological activity mechanisms to contribute to a force to improve the utilization value of soybean protein.

\section{Introduction}

The results showed that soybean protein was a kind of total protein which could meet the requirement of human essential amino acids. It had a variety of health functions, such as the regulation of blood lipids ${ }^{[1]}$, weight loss ${ }^{[2]}$ and so on, it was an important class of active substances. Soy protein mainly included soy protein powder, soy protein concentrate, soy protein isolate and soy tissue protein ${ }^{[3]}$. Over the past decades, soybean tissue protein had been widely recognized and rapid development as a kind of healthy meat substitute ${ }^{[4]}$. The traditional tissue protein had porous structure, but imitation meat taste of processed products was still insufficient. As consumers requirements continue to improve on food taste and quality, soybean tissue protein was increasingly unable to meet the needs of the market. The successful development of soybean drawing protein had met the new needs of customers. Soybean drawing protein was a new type of food industry material, it was a kind of soybean tissue protein with similar muscle fiber texture after the special craft processing, which was based on soy protein isolate as the main raw material, the protein content was in $60 \% \sim 90 \%$, the proportion of amino acid composition was close to the amino acids needed of the human body, it was easy to absorb and it had very high nutritional value ${ }^{[5]}$. Therefore, soybean drawing protein was widely used in various fields of food processing.

\section{Nutrition and Health Function of Soybean Protein}

\section{(1) Effect of lowering blood lipid}

It had been found that soy protein had the effect of reducing blood lipid. Wang ${ }^{[6]}$ et al researched that soy protein decreased triglyceride levels and triglyceride fatty acid fractional synthesis rate of patients with high cholesterol, he found that soy protein consumption of relatively high levels reduced triglyceride levels of patients with high cholesterol. As a product of soybean 
protein, soybean drawing protein also has the effect of reducing blood lipids. Soybean drawing protein was pure plant protein, no cholesterol and it was a kind of high protein and low fat soybean tissue protein products. Soybean drawing protein can improve the protein content of the products, and it can reduce the content of animal fat and cholesterol, thereby it can reduce the triglyceride levels in blood so as to achieve the hypolipidemic effect ${ }^{[7]}$.

\section{(2) Prevention of cardiovascular and cerebrovascular diseases}

The most important risk factor for cardiovascular disease, hypertension, high cholesterol, diabetes, obesity and smoking ${ }^{[8]}$. In October 1999, the U.S. Food and Drug Administration announced the health declaration on soy can reduce the risk of coronary heart disease, they believed that eating low-fat foods containing 25 grams of soy protein per day can significantly reduce the level of cholesterol caused by coronary heart disease ${ }^{[9]}$. After the extrusion of soybean protein, a kind of fibrous protein similar to muscle fiber was formed. As the soybean drawing protein which was replaced animal protein was processed into a variety of leisure food, due to it did not contain cholesterol, people ate later, it would not increase the level of triglycerides in the blood, which can effectively prevent cardiovascular and cerebrovascular diseases to a certain extent, so it was a modern health food.

\section{(3) Weight loss function}

Obesity was a common chronic metabolic disease, it was because that energy intake was greater than the consumption of energy, excess energy accumulated in the body in the form of fat, therefore it leaded to obesity ${ }^{[10]}$. And the effect of reducing blood lipid of soybean drawing protein had a positive effect on weight loss. In recent years, in China, excessive intake of animal protein resulted in the three high, obese people are increasing. How to change this situation from the diet structure? It is necessary to return to the main vegetable protein-based traditional Chinese diet, plant brushed protein was a good starting point. Soybean drawing protein did not contain cholesterol. In addition, it can affect the absorption and accumulation of fat to achieve weight loss by improving satiety and energy consumption.

\section{(4) Natural antioxidants}

It was understood that soybean drawing protein contained natural antioxidants, as wire drawing protein was added to the meat products, it can play a protective role and reduce the oxidation of rancidity. However, its accuracy and mechanism were not clear. May be due to hydrolysis of soy protein, the antioxidant peptide was produced, thereby inhibited the rancidity of meat products oxidation. Torres-Fuentes ${ }^{[11]}$ et al researched identification and characterization of antioxidant peptides from chickpea protein hydrolysates. The results showed that legumin was a source of antioxidant peptides of high interest for food and pharmaceutical industries to develop new nutraceuticals and functional foods.

\section{Application of Soybean protein in Food}

\section{(1) In the application of vegetarian food}

Due the structure of with muscle fibers, plant drawing protein had a good sense of chewing, the appearance of the product had good stability, no beany flavor, so it was suitable to be applied to all kinds of food and advanced bionic food, such as applied to vegetarian ham, vegetarian hot dogs, chicken, vegetarian fish, plain steak, plain dried meat floss, aquatic products. Yang ${ }^{[12]}$ et al developed the filamentous tissue protein balls. Meatballs were producted with filamentous tissue protein as the main raw material with a small amount of pork, meatballs sensory indicators can achieve the same effect of commercially available meatballs, and they had a good sense of chewing 
and viscoelastic. Fei ${ }^{[13]}$ researched on soy drawing protein ham, she used soybean bran protein and gluten as the main raw material with starch, vegetable oil, seasonings and other accessories, the formula of the soybean protein peptide ham was studied through the single factor experiment and orthogonal experiment. Through the sensory analysis of the soybean drawing protein hamburger ham, it was proved that the drawing protein was a kind of soybean tissue protein with muscle fiber structure. It had good chewing sensation and good oil absorption, rehydration and water absorption. It can replace lean meat and is suitable for all kinds of meat products and advanced bionic food applications.

\section{(2) In the application of intestinal food}

Wang ${ }^{[14]}$ et al used of plant bran tissue protein instead of pig meat and added to the red sausage, Substituting pig meat for $25 \%$, the texture property of red sausage was obviously improved, the protein content in red sausage was increased by $13.22 \%$, and the production cost was reduced. Shi ${ }^{[15]}$ developed soybean drawing protein simulation of smoked ham, this review studied the process of the preparation of soybean brushed protein ham. Shi ${ }^{[16]}$ researched on the application of soybean bran protein in crispy intestine, he made production of crisp sausage with soybean bran protein alternative part of lean meat, the results showed that adding a small amount of soybean bran protein, can improve the protein content, replace lean meat, reduce the cost and improve the nutritional value of the product.

\section{(3) In the application of leisure food}

Soybean drawing protein can be used in convenience food, it can be made with a variety of convenience foods. It can give people satisfaction and provide a good source of protein. Such as making seasoning packets, beef tablets of instant noodles and so on. Because it did not contain cholesterol and avoid some of the hazards animal protein ${ }^{[17]}$.

\section{(4) In the application of meat products}

Because soybean protein had good rehydration, oil absorption, emulsification and water absorption ${ }^{[18]}$. It had strong fiber tension, good flexibility and was easy to taste, it also can replace lean meat in the application of the products. So soybean drawing protein had become research direction on the substitution of part of the lean meat in more and more meat products business.

\section{Conclusions}

Soybean drawing protein was a kind of soybean tissue protein with the structure of muscle fiber, so it had a good sense of chewing and rich nutritional value, now soybean drawing protein was widely used in vegetarian food, snack foods and meat products. However, the current national awareness and penetration rate of soybean drawing protein was not high, the research on the application technology of soybean silk protein was not yet mature in the beverage industry and health care products industry. In future research, the emphasis should be on: 1) High efficiency preparation technology of soybean protein products, it will improve the quality of soybean protein products and increase market competitiveness; 2) Expanding the application range of soybean drawing protein; 3) Determining whether the soybean drawing protein had antioxidant activity and its antioxidant mechanism; 4) Researching on hypolipidemic effect of soybean drawing protein and its component on the mechanism of lipid metabolism. These studies will further enhance the application of soybean drawing protein in food. It will improve the use value of soybean protein and promote the expansion of soybean protein market in China, Also it was of great significance to promote the development of China's vegetable protein industry and the improvement of national nutrition. 


\section{References}

[1] J.F. Li, Z.Q. Jiang, Y Li, J.Y. Xiong. Effect of Soybean Protein on Blood Lipid Regulation in Rats. Modern Preventive Medicine[J]. 40:3002-3008. (2013). In Chinese

[2] J Wu, J.Y. Dong, X Jiang and L.Q. Qin. Effect of Soy Protein Supplementation on Weight Loss: a Meta analysis of randomized controlled trials[J]. Health Research.42: 185-189. (2013). In Chinese

[3] X.G. Zhang, J.F. Lu, Y.J. Wang, Y.M. Cheng. Application of Soybean Protein in Conditioning Meat Products[J]. Meat Research.12: 34-36. (2010). In Chinese

[4] A Macedo-Silva, M Shimokomaki, A.J. Vaz, Y.Y. Yamamoto. Textured Soy Protein Quantification in Commercial Hamburger[J]. Journal of Food Composition and Analysis.14:469-478. (2001)

[5] X.M. Qi. Study on Soybean Wire Drawing Protein Vegetarian[J]. Chinese Condiment. 41: 113-114,127. (2016)

[6] Y Wang, P.J.H. Jones, L.M. Ausman. Soy protein reduces triglyceride levels and triglyceride fatty acid fractional synthesis rate in hypercholesterolemic subjects[J]. Atherosclerosis. 173: 269-275 (2004)

[7] H.Y. Wang. Function of Soybean Tissue Protein and Its Application in Meat Products[J]. Meat industry. 33-34.(2007). In Chinese

[8] D.Y. Hu. Paying Attention to Cholesterol Education: Beware of the Second Wave of CardioCerebrovascular Disease in China[J]. Journal of Clinical. 21:77-78. (2006). In Chinese

[9] Y.J Zhao. Provenive plant-derived silk protein: soy protein from Taiwan. Food Safety Guide[J]. 49-50. (2009). In Chinese

[10] X Wang. Discussion on the causes of obesity. The 4th Health Communication Conference Excellent Proceedings[J]. (2009). In Chinese

[11] C Torres-Fuentes, M.M. Contreras, I Recio, M Alaiz. Identification and characterization of antioxidant peptides from chickpea protein hydrolysates[J]. Food Chemistry. 194-202, 2015

[12] Q.Y. Yang, Y Liu. Preparation of filamentous tissue protein. Science and Technology of Food Industry[J]. 28:178-180. (2007). In Chinese

[13] Y.M. Fei. Study on Soybean Bran Protein Ham," Chinese Condiment[J]. 35:40-43. (2010).

[14] S.Y. Wang, Y.M. Fei. Application of plant brushed tissue protein in sausage[J]. Soybean Science and Technology. 4:54-55. (2011). In Chinese

[15]W.T. Shi. Development of Simulated Smoked Ham with Soybean Brushed Protein[J]. Food Research and Development. 33: 98-100. (2012). In Chinese

[16] W.T. Shi. Application of Soybean Protein in Crispy[J]. Food Science and Technology. 38: 120-123. (2013). In Chinese

[17] R.K. Wadhera, M D MPhil, D.L. Steen, I Khan. A review of low-density lipoprotein cholesterol, treatment strategies, and its impact on cardiovascular disease morbidity and mortality[J]. Journal of Clinical Lipidology. 10:472-489. (2016)

[18] L.J. Xie, Y.M. Fei, Y.X. Lv. Functional Properties of Plant Silk Protein and Its Application[J]. Soybean Science and Technology. 35-38. (2011). In Chinese 